\title{
Bibliotecas escolares en prospectiva: el Programa Integral de Bibliotecas Escolares de la provincia de Buenos Aires
}

\author{
Javier Oscar Peón \\ Dirección General de Cultura y Educación. Centro de \\ Documentación e Información Educativa (CENDIE- \\ $D G C y E$ ) / Instituto Superior de Formación Docente y \\ Técnica No 8 / Universidad Nacional de La Plata, \\ Facultad de Humanidades y Ciencias de la Educación, \\ Argentina javierpeon@abc.gob.ar
}

Prospective school libraries: the School Libraries Integral Program of Buenos Aires province

\section{Resumen:}

Se presenta un recorrido por las principales líneas de política pública dirigida a bibliotecas escolares en Argentina, para centrarse en el desarrollo de la experiencia de trabajo del Programa Integral de Bibliotecas de la provincia de Buenos Aires. Las reflexiones sobre los puntos nodales de dicha política, se articulan con el planteo sobre los debates y desafíos que se presentan, para continuar fortaleciendo las bibliotecas escolares y profesionalizando a sus bibliotecarias y bibliotecarios.

Palabras clave: Políticas públicas, Programa Integral de Bibliotecas, Educación Bibliotecas Escolares, Provincia de Buenos Aires, Argentina.

\section{ABSTRACT:}

Main public policy lines aimed at school libraries in Argentina are analyzed, to focus in the development of the work experience of the School Libraries Integral Program of Buenos Aires province. Reflections on core points of such policy are articulated with thoughts about the debates and challenges that arise, in order to continue strengthening school libraries and professionalizing their librarians.

KEYWORDS: Public policies, Integral Libraries Program, Education, School Libraries, Buenos Aires province, Argentina.

\section{UNA BREVE HISTORIZACIÓN DE LAS DECISIONES LEGISLATIVAS Y DE POLÍTICA EDUCATIVA PARA LAS BIBLIOTECAS ESCOLARES}

Pensar el desarrollo y fortalecimiento de las bibliotecas de instituciones educativas como parte de una política pública, implica reconocerlas como agentes activos dentro de la arena política y del gobierno de la educación. En Argentina, las bibliotecas escolares son parte de la trama histórica que se ha ido tejiendo desde la promulgación, en el año 1884, de la Ley Nacional No 1.420 de educación común, laica, gratuita y obligatoria, que a través de la decisión de vincular escuelas con bibliotecas populares, propone satisfacer el acceso a los libros y la lectura.

A nivel de la provincia de Buenos Aires, encontramos que:

Los inicios de las bibliotecas escolares de la Provincia de Buenos Aires se pueden fijar en el período 1875-1898 [...] Las principales líneas de trabajo acerca de las bibliotecas en las escuelas se generan en 1875 y se visualizan, en parte, en el Reglamento General para las Escuelas Comunes de la Provincia de Buenos Aires, sancionado por el Consejo General de Educación el 4 de noviembre de 1876 (CENDIE, 2005, p. 4). 
Vemos que estos son los primeros documentos oficiales de la política educativa destinados a organizar servicios bibliotecarios vinculados al campo de la educación. Luego de ello, no se observa en la legislación la mención explícita de las bibliotecas escolares como parte de un proyecto político educativo.

En la década del '90 se produjo en el campo educativo un fuerte proceso de transformación, con la promulgación de la Ley Federal de Educación $\mathrm{N}^{\circ} 24.195$ (1993), que proponía mejorar la calidad educativa a través de una efectiva equidad, con la renovación de los contenidos curriculares adecuados a los diferentes ciclos y niveles; la evaluación permanente y la formación continua de los docentes. Lo que se presentó como una ola de innovaciones, en realidad significó un vaciamiento de contenidos agravado por fuertes procesos de fragmentación del sistema educativo.

En lo que respecta a dicha ley, en sus capítulos y artículos no se hace mención explícita en ningún caso a las bibliotecas; solo un análisis riguroso de algunos de sus artículos permite, con mucha creatividad e imaginación, forzar la vinculación con el ámbito de las bibliotecas escolares.

Se torna diferente la situación a nivel de provincia de Buenos Aires, ya que la sanción en el año 1993 de la Ley de Educación Provincial № 11.612, establece en el Capítulo IX, en el artículo 28, inciso d: "Crear, subsidiar y sostener bibliotecas, pinacotecas, centros de estudios y entidades culturales" (16).. A partir de ese año se desarrollaron diferentes programas y proyectos nacionales que impactaron en las bibliotecas de los establecimientos de la provincia: "Programa Nacional más y mejor educación para todos", reconocido en nuestra provincia como Plan Social; PRISE (Programa de Reforma e Inversiones del Sector Educación); PRODYMES, entre otros. (Welschen, 2006, p. 3).

A nivel nacional, recién en el año 2006 se sancionó la Ley Nacional de Educación (Ley 26.206), en la cual las bibliotecas escolares son reconocidas en la normativa. Esta ley, promulgada durante el gobierno de Néstor Kirchner, establece el marco legal para promover una política pública de lectura y bibliotecas escolares. En en su Capítulo II, artículo 91, afirma que:

El Ministerio de Educación, Ciencia y Tecnología, en acuerdo con el Consejo Federal de Educación, fortalecerá las bibliotecas escolares existentes y asegurará su creación y adecuado funcionamiento en aquellos establecimientos que carezcan de las mismas. Asimismo, implementará planes y programas permanentes de promoción del libro y la lectura (Ley 26.206, 2006, p. 19).

En el año 2007, la provincia de Buenos Aires sanciona su propia Ley de Educación No 13.688, en línea directa con las definiciones expuestas en la ley Nacional. En su Capítulo IV, Artículo 69, expresa: “Al Director General de Cultura y Educación le corresponden las siguientes competencias [...] Inciso O. Fortalecer las bibliotecas escolares y especializadas existentes y propiciar la creación y adecuado funcionamiento en aquellos establecimientos que carezcan de las mismas".

Podemos afirmar que este paquete de decisiones es un punto de inflexión ya que brinda un renovado marco de referencia para las bibliotecas del sistema educativo, tanto a nivel nacional como provincial.

En este sentido, un caso testigo de acciones que se desprenden de estas políticas públicas y su traducción en acciones legales, es la experiencia de trabajo del programa Integral de bibliotecas escolares en la provincia de Buenos Aires. Con más de 15 años de trayectoria ininterrumpida, el Programa nos permite poner en el centro del debate los desafíos actuales que afrontan las bibliotecas escolares.

\section{Algunos hitos fundantes del Programa Integral de Bibliotecas}

Luego de un largo periodo de ausencia estatal respecto al desarrollo de planes y/o programas destinados a bibliotecarias y bibliotecarios en instituciones educativas, comienza a tomar forma una alianza estratégica entre la Dirección General de Cultura y Educación -DGCyE- de la provincia de Buenos Aires y la Biblioteca Nacional de Maestros -BNM- (2011). Esta alianza está basada en la cooperación y el vínculo interinstitucional que se gesta entre 1998 y 2002 a partir de la definición de líneas de trabajo, que tenían 
la mirada puesta en la modernización de las bibliotecas escolares y la actualización profesional de sus bibliotecarias y bibliotecarios.

El Centro de Documentación e Información Educativa -CENDIE- (actualmente dependiente de la Subsecretaría de Planeamiento) de la DGCyE, se convierte en el agente de desarrollo de políticas para las bibliotecas escolares. El 13 de abril de 2003 se firma el Convenio Marco No 17 (Resolución 1.173) entre la DGCyE y el Ministerio de Educación Ciencia y Tecnología, específicamente la BNM, en el que se expresa:

su deseo de cooperar recíprocamente en la programación de actividades educativas que posibiliten el desarrollo de la gestión de la información en Bibliotecas Escolares y Especializadas del Sistema educativo de la jurisdicción, en los Centros Provinciales de Documentación e Información Educativa y las Bibliotecas Provinciales del Magisterio y/o Pedagógicas a través de distintas acciones de desarrollo de software, capacitación de capacitadores, asistencia técnico-bibliotecológica y otros proyectos que se deriven del presente (Convenio MECT y T N³3, 2003).

Con ese antecedente, la DGCyE firma la Resolución No 860/04 y aprueba el Programa Integral de Bibliotecas del Sistema Educativo de la Provincia de Buenos Aires. Es importante señalar la relevancia de dicho Programa en un enclave geográfico complejo como la provincia de Buenos Aires, con su profunda extensión geográfica y densidad demográfica que ha llevado a la conformación de 25 regiones educativas que abarcan 135 distritos.

Los objetivos y propósitos del Programa, que se fueron plasmando entre los años 2004 y 2019 en los distintos actos resolutivos ministeriales, se orientaron a dar respuesta a las problemáticas propias del quehacer bibliotecario (Peón, 2020).

Operativamente el Programa funciona a partir del trabajo que desarrolla un grupo de bibliotecarias y bibliotecarios referentes (en adelante, BRC) del CENDIE a los cuales se concibe como "el CENDIE en territorio", quienes tienen la función de planificar y desarrollar proyectos con un claro posicionamiento desde una perspectiva regional y distrital. Dicha propuesta busca que desde la biblioteca se impacte en la dinámica escolar, a partir de potenciar con variados recursos informativos el trabajo áulico de docentes, el acompañamiento de las trayectorias educativas de las y los estudiantes, y la contribución al desarrollo sociocultural de la comunidad educativa.

Estos lineamientos generales del Programa toman cuerpo a partir de las acciones propias del rol que cumplen las y los BRC y que sintetizan en asistencias técnicas y reuniones con bibliotecarios para la orientación en el desarrollo de proyectos de elaboración del manual de procedimientos de las bibliotecas; el acompañamiento a los bibliotecarios en la elaboración del plan institucional de biblioteca en el marco del proyecto institucional de la escuela; el trabajo conjunto con el Plan Provincial de Lectura y Escritura (Res. 1.080/17) para la realización de actividades de promoción de la lectura en las escuelas — tales como rondas de narración, maratones de lectura y caminatas por la lectura-. También se visualizan estos lineamientos en la organización de Jornadas Distritales, Regionales e Interregionales de Bibliotecarios Escolares para promover su actualización profesional, profundizando el diálogo sobre las heterogéneas realidades de la provincia, a la vez que intercambiar ideas, proyectos, saberes; y acompañar las actividades que bibliotecarias y bibliotecarios realizan en las regiones, proyectándolas a través de su difusión en las redes sociales que las y los BRC administran.

No podemos dejar de mencionar que la DGCyE, en el marco de una nueva gestión de gobierno, asumió la decisión política de dar continuidad del Programa, atravesada por un contexto mundial inédito de pandemia por COVID-19 que en Argentina se inicia en los primeros meses de 2020 y aún continúa.

Considerada por las máximas autoridades del ámbito educativo provincial, como una política institucional histórica del CENDIE, se aprueba la continuidad del Programa Integral de Bibliotecas Escolares y Especializadas del Sistema Educativo de la provincia de Buenos Aires, por acto resolutivo (Res. 1.501/20).

Este nuevo lanzamiento del Programa redefine sus propósitos, los cuales se orientan el diseño y ejecución de proyectos y acciones para fortalecer la dinámica de funcionamiento de las bibliotecas del sistema educativo provincial, propiciar la articulación y la comunicación entre BRC de regiones y distritos, generar estrategias 
de trabajo colaborativo que fortalezcan el rol de las bibliotecarias y los bibliotecarios promoviendo en el acceso igualitario de sus usuarias y usuarios a la información y el conocimiento.

A su vez, el Programa expone una serie de objetivos tales como: mejorar la administración de los recursos y servicios y la gestión de información, promover prácticas de lecturas diversas y plurales, propender a la formación de usuarias y usuarios lectoras y lectores en múltiples formatos y soportes; incentivar el desarrollo de actividades de extensión bibliotecaria y de extensión cultural favoreciendo el contacto con la comunidad educativa; acentuar el carácter pedagógico del rol y las funciones que desarrollan las bibliotecarias y los bibliotecarios, junto a los equipos directivos; implementar proyectos vinculados a la recuperación, conservación y difusión de la memoria histórica de las instituciones educativas; y construir redes reales y virtuales de información y comunicación.

Podemos destacar un punto de interés en este renovado impulso del programa: la importancia de desarrollar propuestas de trabajo en articulación con las direcciones de los niveles educativos y con el Plan Provincial de Lecturas y Escrituras dependientes de la DGCyE.

No hemos tenido la pretensión de realizar una descripción exhaustiva de Programa Integral, sino de mostrar sus elementos fundantes, sus aspectos estructurales y sus perspectivas de trabajo actuales, puesto que nuestro propósito fundamental aquí es posar la mirada sobre algunos desafíos a los que se enfrentan hoy las políticas para las bibliotecas escolares y sus bibliotecarias y bibliotecarios, y a los que en el ámbito provincial, las finalidades y objetivos del programa integral intentan dar marcos de referencia y de acompañamiento para proyectar en el tiempo su concreción progresiva.

\section{Desafíos actuales de las políticas públicas para Fortalecer a las bibliotecas DE INSTITUCIONES EDUCATIVAS}

Se hace necesario pensar no solo la responsabilidad de las políticas públicas en el fortalecimiento de las bibliotecas de instituciones educativas, sino también debatir sobre el modelo de biblioteca escolar que se quiere lograr en cada escuela, sin dejar de pensarlo a la luz del proyecto político pedagógico que ésta sustente. Son las condiciones materiales, sociales y culturales sobre las que funciona cada institución educativa, las que de una u otra manera influirán en la definición del modelo de biblioteca para su comunidad. Y ese modelo debe ser un proceso de construcción sobre la base de la reflexión, el debate y el logro de acuerdos por parte de todas y todos sus integrantes.

Dicho esto, nos proponemos desplegar brevemente algunos desafíos que las políticas públicas sobre bibliotecas escolares dejan entrever en sus acciones y resultados de gestión, y que será importante abordar desde una mirada prospectiva.

A modo de una bitácora que posibilita el registro de la experiencia de trabajo, sus aproximaciones en la búsqueda de nuevas soluciones y su socialización con la comunidad de especialistas para su discusión, exponemos aquí esos puntos clave.

Punto 1. Gestión de la biblioteca escolar.

La planificación de la biblioteca es un componente fundamental para su funcionamiento en tanto nos permite contribuir a la gestión de una biblioteca escolar de acuerdo con las necesidades de información de sus usuarias y usuarios, potenciar los servicios y las colecciones según el nivel y/o la modalidad de escolarización, formalizar los procesos técnicos y sus procedimientos. Todo lo cual favorece los procesos de enseñanza y de aprendizaje y desempeño de sus docentes así como progreso cultural de sus estudiantes.

A su vez, gestionar los materiales que conforman la colección de una biblioteca debe responder a estrategias que faciliten el acceso a todas las formas de registro documental, que promuevan el derecho a la lectura y el encuentro de experiencias compartidas como comunidad de lectoras y lectores. 
En sintonía con las ideas de Castán Lanaspa (2008), pretendemos acercarnos a un modelo de biblioteca que, como la escuela en su conjunto, se esfuerce por prestar un buen servicio de apoyo a la escolaridad de todos sus integrantes, y tenga la sensibilidad de dedicar una parte de sus desvelos, sus recursos y su tiempo a dar o ampliar, aunque sea muy modestamente, las posibilidades de los que ya tienen muy pocas.

Punto 2. Promoción y mediación a la lectura.

Las bibliotecas en las instituciones educativas cumplen un rol preponderante en los procesos de transmisión y apropiación del universo cultural por parte de sus estudiantes. Las bibliotecarias y los bibliotecarios deben ser mediadores activos de múltiples prácticas lectoras, la integración de las diversas experiencias de interacción con las lecturas, simplemente por el placer de leer teniendo acceso a un universo cultural amplio; pero también propiciar formas de comprensión lectora que faciliten el estudio, la investigación y el acceso la producción de conocimientos.

Punto 3. Tecnologías.

Reconocemos que el desarrollo, acceso y utilización de las tecnologías en la biblioteca escolar se presenta como el punto que mayores debates suscita y es unos de los más complejos de analizar en el contexto contemporáneo. Por un lado, las tecnologías han pasado a formar parte de la vida cotidiana de la ciudadanía; una inagotable bibliografía al respecto asume que las bibliotecas escolares requieren de su presencia tanto para el trabajo bibliotecológico como para el despliegue de estrategias de atención de sus usuarias y usuarios, principalmente vinculadas a las alfabetizaciones múltiples (informacional, tecnológica, digital, audiovisual). Por otro lado según el último censo realizado por el CENDIE (2012), muestra que en la provincia de Buenos Aires, un 59\% de escuelas poseen biblioteca, y que el 50\% de éstas posee al menos una computadora que se prioriza para el trabajo del profesional de la biblioteca.

Más allá de los planes y programas que promueven el acceso a tecnologías desde el ámbito público, desandar este sinuoso camino será uno de los desafíos venideros, al cual se suma la brecha en el acceso y uso de las tecnologías y acceso a internet, que puso al descubierto la pandemia, para el conjunto de la comunidad educativa.

Aquí mencionamos solo algunos factores tecnológicos necesarios para las bibliotecas escolares que podrán orientan la discusión, entre ellos: la utilización de software libre para la gestión integral de la biblioteca, selección de recursos digitales y electrónicos (curación de contenidos) y creación de espacios de acceso abierto a la información educativa, difusión de la biblioteca en redes sociales, entre muchos otros.

Punto 4. Vínculos con la comunidad educativa.

Las bibliotecas históricamente han sido espacios más restringidos en su acceso, y como sabemos, desde las últimas décadas del siglo XX han comenzado a considerar la necesidad de abrirse a la comunidad e intentar captar su atención y a los usuarios.

Las estrategias con las que la biblioteca se abre a la comunidad permiten la realización de proyectos culturales y socio comunitarios, que sensibilicen al campo educativo para la participación en actividades compartidas y la consolidación del vínculo entre la escuela y las familias en torno a las prácticas de lectura, el acceso a la información y el conocimiento a través de sus colecciones y servicios.

Los vínculos no solo serán con la comunidad educativa sino que una importancia creciente reviste para bibliotecarias y bibliotecarios la participación activa en redes de bibliotecas escolares, a partir de un plan de cooperación y trabajo mutuo, compartiendo información y recursos con la finalidad de potenciar y enriquecer las estrategias de trabajo en pos de dar centralidad a la biblioteca en la institución educativa y su comunidad de usuarias y usuarios.

Punto 5. Profesionalización y actualización 
Es tarea de bibliotecarias y bibliotecarios, velar por el fortalecimiento y visibilidad del espacio de la biblioteca, trabajar a favor de ese objetivo desde el lugar de cada uno y cumpliendo con las coresponsabilidades específicas del rol que se desempeña. El retiro o la ausencia de nuestra función como profesionales no puede ser relegada bajo ninguna circunstancia. El Reglamento de instituciones educativas de la provincia de Buenos Aires (Dirección..., 2012), establece los derechos y obligaciones de bibliotecarias y bibliotecarios en la institución educativa, y en el Artículo 78 inciso 5 establece: "Cumplir las tareas que se le asignen en el marco del Proyecto Institucional y en tanto guarden relación con la naturaleza y competencia de su cargo y se funden en razones de promoción de derechos de los alumnos".

Los siguientes ejes para el debate, vinculados a la profesionalización y actualización, son el fundamento para que los cuatro puntos anteriores se constituyan en los lineamientos que deberán atender las políticas públicas para el ámbito de las bibliotecas escolares y sus profesionales.

Consideramos que la actualización y profesionalización deberá construirse y sostenerse en el conocimiento de la normativa que emana de la política educativa en general y para las bibliotecas escolares en particular. A su vez, la formación permanente a través de la participación en acciones de capacitación de la especialidad o afines a ella facilitará la actualización profesional contribuyendo a mejorar las condiciones de trabajo y el funcionamiento del espacio de la biblioteca.

Por último, fomentar el desarrollo de experiencias de investigación sobre la biblioteca escolar como objeto de estudio con múltiples vertientes, para ello la metodología de investigación-acción puede ser de gran ayuda para iniciar el recorrido. Este eje tiene fuerte vinculación con un aspecto que es necesario conquistar para robustecer nuestra profesión, hacer un salto cualitativo que nos permita transformar nuestro quehacer cotidiano en una praxis bibliotecológica, es decir, una práctica reflexiva y crítica, dialogada, debatida y mejorada a partir de la mirada de otras y otros colegas, docentes, etc. Pero no solo eso, sino que es necesario algo más, ser productores y escritores, esto es, bibliotecarias y bibliotecarios como autores que registran y narran pedagógicamente sus experiencias bibliotecológicas. Para ello será necesario adentrarnos en una forma de escritura académica que pula nuestras ideas para sistematizar, publicar y difundir esas narrativas a la comunidad de especialistas, a la vez que, al documentarlas, hacerlas parte de la memoria de la institución educativa.

\section{A MODO DE COROLARIO}

Sabemos del compromiso de bibliotecarias y bibliotecarios en el desempeño profesional que llevan adelante y del valor de lo que la biblioteca significa para el sostenimiento de las trayectorias educativas de nuestras y nuestros estudiantes.

Estos desafíos aquí planteados, que son producto de las reflexiones y debates en el marco de la experiencia de trabajo y resultados que año a año van surgiendo en el marco del programa integral, no pretenden ser exhaustivos ni constituyen una propuesta cerrada, sino, muy por el contrario, una descripción de aspectos nodales que se presentan para su problematización y debate.

Se deberán constituir acuerdos y consensos para planificar políticas, planes, programas y proyectos en años venideros, donde otros imaginarios comiencen a construirse, instalando otras palabras, otros discursos y otras prácticas, acordes con una perspectiva del derecho social a la educación.

Es menester dejar en claro las bibliotecas escolares tienen como misión intervenir para mejorar las prácticas educativas, y sus bibliotecarias y bibliotecarios son mediadores fundamentales desde su especificidad, fortaleciendo los procesos de enseñanza y aprendizaje, sosteniendo las trayectorias de las y los estudiantes, que no solo son de tal o cual institución educativa, sino que son de todas y de todos.

Acompañarlos y cuidarlos, ese es y será nuestro mayor desafío. 


\section{REFERENCIAS}

Biblioteca Nacional de Maestros. (2011). Programa Bibliotecas Escolares y Especializadas de la República Argentina. Una política pública para las Bibliotecas del sistema educativo. Ciudad Autónoma de Buenos Aires: Biblioteca Nacional de Maestros. Recuperado de http://www.bnm.me.gov.ar/redes_federales/publicaciones/doc/coleccio n_rf_cuadernillo-1.pdf

Castán Lanaspa, G. (2008). La biblioteca escolar y la igualación de oportunidades. En: Bonilla, E., Goldin, D., y Salaberria, R. (coords.) Bibliotecas y escuelas: retos y posibilidades en la sociedad del conocimiento (pp. 77-107). México: Océano.

CENDIE. Centro de Documentación e Información Educativa. (2012). Situación de las bibliotecas escolares bonaerenses. Recuperado de http://servicios.abc.gov.ar/lainstitucion/organismos/cendie/censo_biblioteca_200 9/censo_bibliotecas_2009.pdf

CENDIE. Centro de Documentación e Información Educativa. (2003). Aguapey en la provincia. Una propuesta informática para el nuevo escenario de las Biblioteca Escolar, una mirada desde su pasado. Biblio XXI, 1(1).

CENDIE. Centro de Documentación e Información Educativa. (2005). Programa Integral de Bibliotecas. La formación y la capacitación permanente se transforman en acciones prioritarias, para el desarrollo de habilidades para la selección de la información. Biblio XXI, 4(4).

Dirección General de Cultura y Educación. (2012). Reglamento General de las Instituciones Educativas de la Provincia de Buenos Aires. Recuperado de http://servicios.abc.gov.ar/lainstitucion/organismos/consejogeneral/reglamen to_general/reglamento_general_de_las_instituciones_educativas.pdf

Ley 11.612. Ley de Educación de la Provincia de Buenos Aires. 23 de diciembre de 1995. Recuperado de https://www. argentina.gob.ar/normativa/provincial/ley-11612-123456789-0abc-defg-216-1100bvorpyel

Ley 13.688. Boletín Oficial de la Provincia de Buenos Aires, 7 de julio de 2007. Recuperado de https://normas.gba. gob.ar/ar-b/ley/2007/13688/3181\#

Ley 24.195. Sistema educativo nacional. Ley Federal de Educación, 5 de mayo de 1993. Recuperado de http://servici os.infoleg.gob.ar/infolegInternet/verNorma.do?id=17009

Ley No 26.206. Boletín Oficial de la República Argentina. 14 de diciembre de 2006. Recuperado de https://www.arg entina.gob.ar/sites/default/files/ley-de-educ-nac-58ac89392ea4c.pdf

Peón, J. O. (2020). El Programa Integral de Bibliotecas Escolares y especializadas del sistema educativo de la provincia de Buenos Aires: una política innovadora con sello propio. Anales de la educación común, 1(1-2), 221-233. Recuperado de https://cendie.abc.gob.ar/revistas/index.php/revistaanales/article/view/96

Resolución 860. (2004). Programa Integral de Bibliotecas del Sistema Educativo. Recuperado de http://servicios2.abc.gov.ar/lainstitucion/revistacomponents/revista/archivos/anales/numero07/archivosp araimprimir/10_normativas_st.pdf

Resolución No 1.080/17. Plan Provincial de Lectura y Escritura. 9 de mayo de 2017.

Resolución 1.173. (2003). Convenio Marco No 17 entre la Dirección General de Cultura y Educación y el Ministerio de Educación Ciencia y Tecnología.

Resolución 1.501. (2020). Boletín oficial de la Provincia de Buenos Aires, 2 de abril de 2020.

Resolución 2.245 (2009). Boletín oficial de la Provincia de Buenos Aires, 17 de julio de 2009. Recuperado de https:/ /normas.gba.gob.ar/ar-b/resolucion/2009/2245/202832

Welschen, M. (2006). Estado de situación de las bibliotecas y de los bibliotecarios escolares del sistema educativo de la provincia de Buenos Aires. En Jornada de bibliotecas escolares. Reunión Nacional de Bibliotecarios, Asociación de Bibliotecarios Graduados de la República Argentina. Ciudad Autónoma de Buenos Aires, Argentina. 